\title{
Updates in sepsis management: new guidelines for sepsis care
}

Ruth Kleinpell, PhD RN FCCM FAAN, Director, Center for Clinical Research and Scholarship, Rush University Medical Center; Professor, Rush University College of Nursing; Nurse Practitioner, Mercy Hospital \& Medical Center, Chicago, Illinois, USA; President, World Federation of Critical Care Nurses

Key Words: guidelines * intensive care * management * sepsis *

E-mail: Ruth_M_Kleinpell@rush.edu

\begin{abstract}
ESPAÑOL
Actualización en el manejo de la sepsis: nuevos lineamientos para el cuidado de la sepsis

Palabras clave

Cuidado intensivo, lineamientos, manejo, sepsis

Resumen

Este articulo resume los nuevos Lineamientos de la Campaña Sobreviviendo a la Sepsis e identifica las implicancias para la práctica de la enfermeria.
\end{abstract}

\section{SUMMARY}

- This article summarises the new Surviving Sepsis Campaign Guidelines and identifies implications for nursing practice.

\section{INTRODUCTION}

Sepsis is a worldwide healthcare condition that is responsible for a significant percentage of neonatal, pediatric and adult deaths. It occurs as a result of the systemic manifestation of infection. Severe sepsis (sepsis that has progressed to cellular dysfunction and organ damage or evidence of hypoperfusion) and septic shock (sepsis with persistent hypotension despite adequate fluid resuscitation) represent significant stages of sepsis that increase mortality rates for patients.

Improving the recognition of sepsis can help in early detection and treatment to ultimately enhance outcomes for patients. Nurses play an important role in promoting best care practices for patients with sepsis in promoting early identification and in instituting treatment measures.

\section{GUIDELINES}

New evidence based guidelines for the management of sepsis, the Surviving Sepsis Campaign Guidelines, will be published in 2012 and represents the work of a committee of 65 international experts representing 26 international organizations, including The World Federation of Critical Care Nurses (WFCCN). The guidelines use the Grades of Recommendation, Assessment, Development and Evaluation (GRADE) system to establish the quality of evidence from high (A) to very low (D) and to determine the strength of recommendations from strong (2) to weak (1).

\section{GUIDELINE COMPONENTS}

The new Surviving Sepsis Campaign Guidelines update the prior guidelines (Dellinger vet al., 2008), and provide recommendations for the medical management of sepsis including (Dellinger et al., 2012):

- treatment of sepsis-induced hypoperfusion (hypotension persisting after an initial fluid challenge or blood lactate concentration $>4 \mathrm{mmol} / \mathrm{L}$ ) with fluid resuscitation ( $\geq 1000 \mathrm{~mL}$ of crystalloids to achieve a minimum of $30 \mathrm{ml} / \mathrm{kg}$ of crystalloids in the first 4 to 6 hours), guided by perfusion indices (central venous pressure (CVP) $8-12 \mathrm{~mm} \mathrm{Hg}$; mean arterial pressure (MAP) $\geq 65 \mathrm{~mm} \mathrm{Hg}$, urine output $\geq 0.5 \mathrm{~mL} / \mathrm{kg} / \mathrm{hr}$ or central venous (superior vena cava) $>70 \%$ or mixed venous oxygen saturation $\geq 65 \%$;

- blood cultures before antibiotic administration and administration of broad-spectrum antibiotic therapy within one hour of diagnosis of septic shock;

- use of vasopressor therapy (with norepinephrine as a first choice) to target a MAP of $65 \mathrm{~mm} \mathrm{Hg}$;

- low tidal volume ventilation (6 mL/kg (predicted) body weight) and limitation of inspiratory plateau pressure strategy for sepsis-induced acute respiratory distress syndrome.

Other treatment recommendations reinforce the use of stress ulcer prophylaxis, deep vein thrombosis prophylaxis, nutritional considerations with oral or enteral feedings, as tolerated, rather than either complete fasting or provision of only intravenous glucose within the first 48 hours after a diagnosis of severe sepsis/septic shock; sedation and analgesia with the use of either intermittent bolus sedation or continuous infusion sedation with daily interruptions or lightening of sedation; and institution of glycemic control targeting a blood glucose $<150 \mathrm{mg} / \mathrm{dL}$, among other recommendations (Dellinger et al., 2012).

A new area of focus in the guidelines addresses the importance of setting goals of care. Previously labeled as "Consideration for Limitation of Support", the new recommendation for "Setting Goals of Care" focuses on discussion of prognosis with patients and 
families. Goals of care should be addressed as early as feasible but no later than within 72 hours of admission to the ICU. Additionally, the integration of palliative care principles and as appropriate, endof-life care planning is recommended (Dillinger et al., 2012). This new focus advocates for patient and family centered care and discussion of prognosis - a component of care that nurses have long recognized as important for the critically ill patient. Moreover, research has identified that critical care nurses can experience moral distress when caring for patients who have limited prognosis but continue to receive life sustaining therapies (American Association of Critical Care Nurses, 2012). This component of the revised guidelines will help promote family care conferences for communication and discussion of appropriate goals of care and potentially prevent moral distress among ICU caregivers.

\section{NURSING IMPLICATIONS}

A number of strategies can be used to integrate the Surviving Sepsis Campaign Guidelines in nursing practice including promoting awareness of the new guidelines and using the new guidelines as a performance improvement initiative to improve sepsis care for patients (Table 1).

The WFCCN published a nursing companion guide to the Surviving Sepsis Campaign Guidelines in 2011 which outline a number of nursing recommendations for care of the patient with sepsis (Aitken et al., 2011). This publication represented the work of an international taskforce of WFCCN members, led by Professor Leanne Aitken of Australia. The publication is available full text on the WFCCN website to promote dissemination of this key nursing consideration document to improve care for the patient with severe sepsis (http://en.wfccn. org/resources_sepsis.php). A total of sixty-three recommendations related to the nursing care of severe sepsis patients were outlined including prevention measures addressing education, accountability, surveillance of nosocomial infections, hand hygiene, prevention of respiratory, central line-related, surgical site and urinary tract infections, with infection management recommendations focused on both control of the infection source and transmission based precautions (Aitken et al., 2011). Recommendations related to initial resuscitation include improved recognition of the deteriorating patient and initiating early resuscitation measures (Aitken et al., 2011). Additional recommendations advocate for supportive nursing care for nutrition, mouth and eye care, and pressure ulcer prevention and management.

The 2012 International Surviving Sepsis Campaign Guidelines provide a comprehensive review of the medical management of patients with sepsis and septic shock. The guidelines have implications for nursing care, and application of the new recommendations into nursing practice can help ensure competent nursing care to promote optimal outcomes for critically ill patients with sepsis.
- Disseminate information on the new guidelines to members of the ICU team

- Include discussion of the guidelines during unit clinical care meetings and clinical rounds

- Formulate a multidisciplinary team and outline a timeline for implementing the guidelines

- Use the new guidelines as a performance improvement initiative for the ICU as well as noncritical care areas to improve recognition and treatment of patients with sepsis

Specific areas include:

- Aid in the early identification of sepsis including recognizing patients at risk for developing sepsis (patients who are elderly, immunocompromised, patients with surgical/invasive procedures, indwelling catheters, mechanically ventilated patients) and monitoring physical assessment parameters including vital signs and perfusion status (urine output, mental status changes, skin color)

Provide comprehensive sepsis treatment (circulatory support with fluids, inotropes, and vasopressors; supportive treatment with oxygenation and ventilation; antibiotic administration; sepsis guideline recommendation measures; monitor and report patient response to treatment)

- Promote patient and family centered care (patient and family teaching, addressing the needs of families of critically ill patients, setting goals of care, and holding family care conferences to discuss goals of care)

Table 1. Strategies to integrate the Surviving Sepsis Campaign Guidelines in nursing practice

\section{REFERENCES}

American Association of Critical Care Nurses (2012). Moral Distress - Public Policy Position Statement. [Online] Available at: http:// www.aacn.org/WD/practice/Docs/Moral_Distress.pdf Accessed 05 June 2012.

Dellinger RP, Levy ML, Opal S, Gerlach H, Sevransky J, Sprung CL, Townsend S, Kleinpell R, Deutschman C. et al. (2012). Surviving Sepsis Campaign: International guidelines for management of severe sepsis and septic shock. Critical Care Medicine, In Press.

Dellinger RP, Levy ML, Carlet JM, Bion J, Parker MM, et al. (2008). Surviving sepsis campaign: international guidelines for the management of severe sepsis and septic shock: Critical Care Medicine 36: 296-327.

Aitken LM, Williams G, Harvey M, Blot S, Kleinpell R, Labeau S, et al. Nursing considerations to complement the Surviving Sepsis Campaign. Critical Care Medicine 2011; 39:1800-1818. 